\title{
DISTRIBUTION OF METASTASES IN ENT AREA - COMPARISON OF THEORY AND PRACTICE
}

\author{
Behanova E, Pedan H, Hanzel P, hajtman A, Calkovsky V. \\ Clinic of Otorhinolaryngology and Head and Neck Surgery, Jessenius Faculty of Medicine in Martin, \\ Comenius University in Bratislava, and Martin University Hospital, Martin, Slovakia
}

\begin{abstract}
A b s tract
In head and neck cancers the occurrence of nodal metastases is the most important prognostic factor. Their early diagnosis is crucial for proper treatment. Detection of early metastases is still very difficult. Predictive diagnostic methods such as the sentinel lymph node detection is limited by the occurrence of skip metastases. At our Clinic we prefer a selective neck dissection based on a surgical treatment of predilected lymphatic spread area for each type of head and neck tumor with a preservation of non-lymphatic structures of the neck. The main objective of this article is to analyze the distribution of neck metastases and to study the frequency of skip metastases in head and neck cancer.
\end{abstract}

Key words: skip metastasis, head and neck cancer, lymph node dissection

\section{INTRODUCTION}

Metastasis is a secondary malignant tumor which is formed by breaking away from the primary cancer and spreading through blood, lymph system, or by direct spread to the surroundings, and form new metastatic tumors in other parts of the body. The ability to metastasize is one of the essential signs of malignancy $(1,2)$.

One of the most important prognostic factor in head and neck cancers is the occurrence of nodal metastases (1). The incidence of neck metastases (MTS) reduces patients overall survival by almost $50 \%$ and increases the risk of disease recurrence (3). Only a smaller per cent of oncologic patients die as a result of a size of the primary tumor, the death is most often caused by metastases (2). Early diagnosis and identification of the exact location of MTS is crucial for choosing the right surgical treatment $(1,4)$.

Metastases in the neck are manifested mostly by solid, slow-growing swelling, the skin above is intact, without local inflammation. Initially, the nodes are movable towards the skin and the base, gradually occurs infiltration of the surrounding structures and fixation of the tumor. Nodal metastases are usually unilateral, bilateral occurrence is typical in malignancies of the tongue root and nasopharynx or indicates extension of the primary tumor to both sides or a generalization of the disease. Characteristic sign in carcinomas of the tonsils and the tongue root are cystic metastases in the jugulodigastric region. In advanced metastases we can observe necrotic and inflammatory changes in lymph nodes with infiltration of surrounding structures $(1,5,6)$.

Other associated symptoms such as dysphagia, odynophagia, otalgia, dysphonia, or dyspnoea are determined by the primary tumor. General non-specific symptoms include increased fatigue, anorexia, and cachexia (2).

Corresponding author: Pavel Hanzel, MD, PhD., e-mail: pavelhanzel@gmail.com

(C) 2021 Hanzel P. et al.

This work is licensed under the Creative Commons Attribution-NonCommercial-NoDerivs 4.0 License (https://creativecommons.org/licenses/by-nc-nd/4.0/) 
Based on the location of the primary tumor we can predict the probable spread of metastatic disease to lymph nodes. According to the American Academy of Otolaryngology

Head and Neck Surgery classification, also known as Robbins classification, lymph nodes of the neck are divided into 6 areas $(1,3)$.

Tumors of the nasopharynx have a high incidence of metastases regardless of the stage of the disease. They occur in $70-80 \%$ of patients in areas II and V (1).

The oropharyngeal region is one with the largest lymphatic supply, therefore, at the time of diagnosis, up to $75 \%$ of patients have established nodal metastases.

In hypopharyngeal tumors metastases are a little less frequent - 60\% (5, 6, 9).The most often affected are the ipsilateral deep jugular nodes in areas II, III, and IV. If the tumor extends beyond the midline, metastases may be bilateral - e.g. in tongue root or soft palate cancer $(1,5,9)$.

Tumors of the larynx are one of the most common malignancies in head and neck, accounting for approximately 25-30\% (4). In supraglottic and infraglottic tumors the incidence of MTS is 35-45\%, similar to the oropharyngeal region, they have large lymphatic supply, so bilateral nodal involvement is not uncommon. In contrast, glottic carcinomas, due to a weak lymphatic supply, metastasize rarely, only in advanced stage of the disease. Laryngeal tumors typically metastasize to areas II, III, and less commonly to areas IV and VI. The spread of MTS in areas I and V is exceptional and does not occur without the simultaneous presence of MTS in areas II and III $(1,4,5)$.

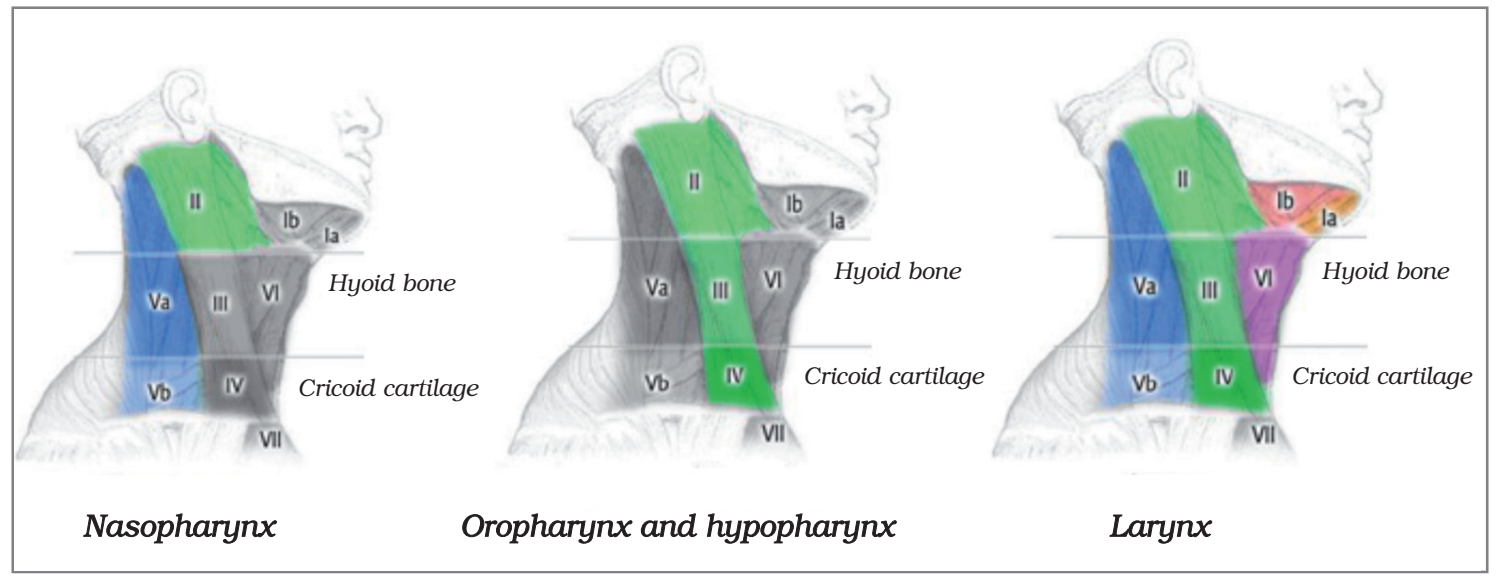

Fig. 1 Metastatic spread according to the location of primary tumor (10)

An early detection of nodal metastases remains a major challenge despite the constant development of imaging modalities (7). Commonly available screening methods help us to detect approximately 70-80\% of metastases, while small metastatic nodes without necrosis and clinically obvious symptomatology remain undetectable in 10-70\% of patients (3). Regarding this fact, radical, elective neck dissections have been performed in the past to detect occult metastases before their clinical manifestation (1). As a result, approximately $60-70 \%$ of patients were overtreated, as they underwent redundant, invasive procedures with significant associated complications (7).

Therefore, it was necessary to develop a predictive diagnostic method that can increase diagnostic accuracy, including negative predictive value, enough to reduce unnecessary elective dissections of the neck.

Sentinel lymph node (SLN) detection and biopsy was considered to be such a method. SLN is defined as the first node into which lymph enters from the primary tumor. The aim of the biopsy is to detect occult metastases - if the SLN is invaded by malignancy, the spread of 
the disease to other descending nodes is highly probable and block neck dissection is indicated (8).

However, the limiting factor of this method is the occurrence of skip metastases. This term is used when a tumor cell skips the nearest descending lymph node and forms a metastasis in the next node.

The occurrence of skip metastases has been confirmed by several retrospective studies. Shikharani and colleagues reported a 6.7\% incidence of skip metastases in a group of 30 patients with oral cancer (9).

In recent years, positron emission tomography (PET) has been increasingly used. It is a functional imaging technique that uses radiotracers to visualize and measure

metabolic changes in tissue. In detection of malignant lymph nodes, PET-CT has 90\% sensitivity and 94\% specificity (1). Currently, one of the leading methods is dual-source multienergy CT with texture analysis (7).

The aim of our work is to analyze the distribution of neck metastases and to study the frequency of skip MTS in head and neck cancer.

\section{METHODS}

At our Clinic we prefer a selective neck dissection based on a surgical treatment of predilected lymphatic spread area for each type of head and neck tumor individually with a preservation of non-lymphatic structures of the neck. In case of a negative - NO clinical finding on the neck we use perioperative biopsy of lymph node in a predilected area. When the biopsy is negative we continue with a selective neck dissection only of the predilected lymphatic area. When the biopsy is positive, or in case of a clinically apparent metastatic disease, we continue with a selective neck dissection of levels I-V. If the primary tumor extends beyond the midline, or in an advanced stage of the disease (clinically bilateral $\mathrm{N}^{+}$ finding), we approach to a bilateral selective neck dissection.

The surface size of a tumor does not correlate with the incidence of metastasis, therefore, the consideration of the patient's T category might not be sufficient for making optimal treatment decisions $(11,12)$.

In our retrospective study within period from 1st January 2015 to 31th December 2019 we evaluated 396 patients who had been treated at our Clinic for an oncologic disease. In this group of patients we evaluate the location of the primary tumor, the type of block neck dissection, the separation quality of individual areas of lymphatic tissue, and the histopathology report.

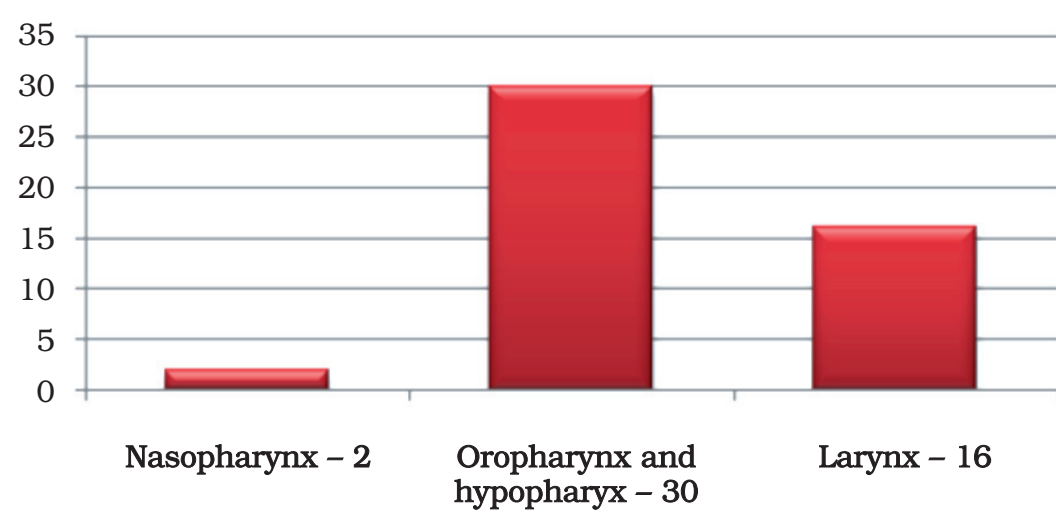

Fig. 2 Group of patients in study 
48 patients were included in the study, in which a neck block dissection was performed with a thorough separation of adipo-lymphatic tissue according to the neck levels I-V, and a nodal metastasis of the primary tumor was confirmed.

The remaining 348 patients were excluded from the study due to a negative (NO) histopathology report or because of the inability to determine exactly in which group the metastatic lymph nodes were present due to an insufficient lymphatic tissue separation during the surgery.

\section{RESULTS}

The study population comprised of 45 males $(93,7 \%)$ and 3 females $(6,2 \%)$. The age of the patients ranges from 18-90 years. In 34 cases $(70,8 \%)$ nodal metastases were clinically apparent or highly suspected, in the rest of them $(29,2 \%)$ there was NO finding.

1) In the group with nasopharyngeal cancer we had 2 patients, in both cases MTS were confirmed in the predicted area II. In addition, both had a nodal metastasis in area III, too.

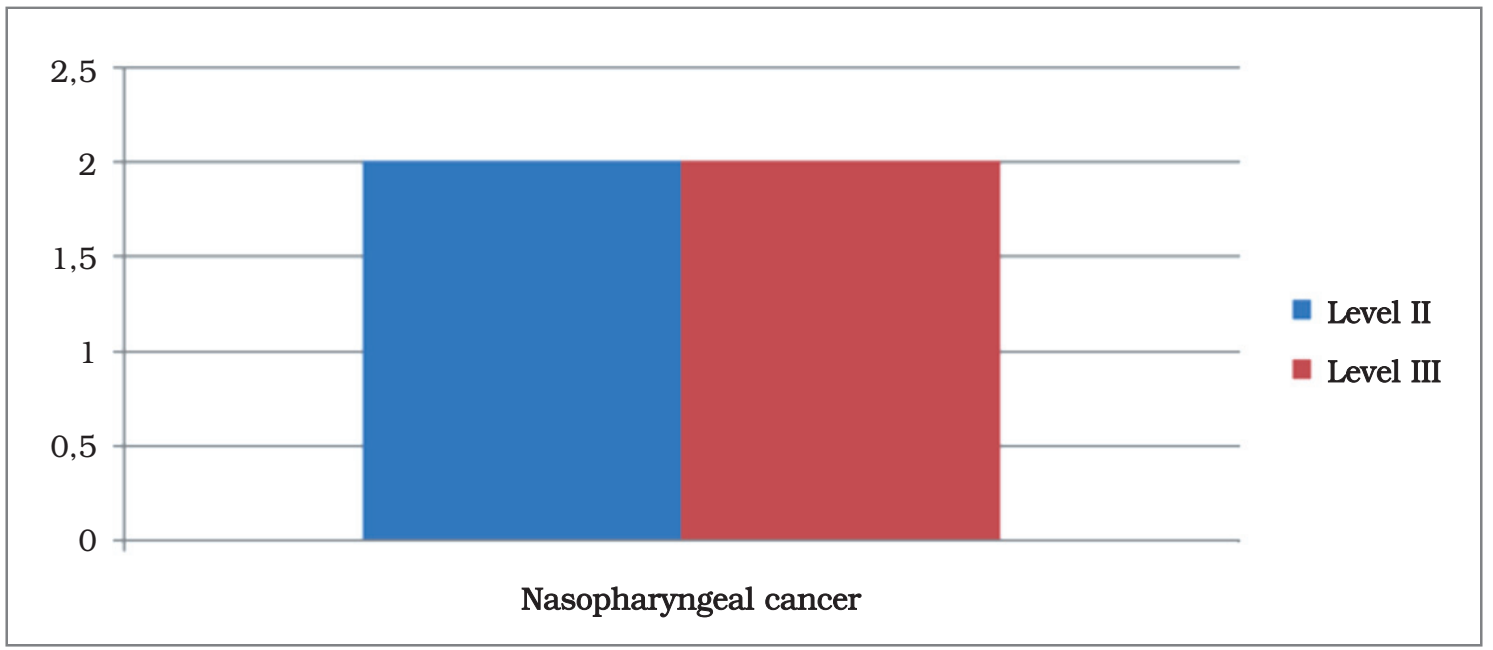

Fig. 3 Nodal MTS in nasopharyngeal cancer

2) We had 30 patients with confirmed MTS of oropharyngeal and hypopharyngeal tumors. 29 patients had a histologically verified metastasis in the predilected area II, III, or IV. Two of these patients had also a MTS in the area V. Additionally, 3 patients - 2 with carcinoma of the tongue root and 1 with carcinoma of the uvula had MTS bilaterally, which also corresponds to the data from literature.

Differences in the distribution of MTS were observed in 1 patient with a tongue root carcinoma in whom a MTS was histologically verified in the level Ib. 


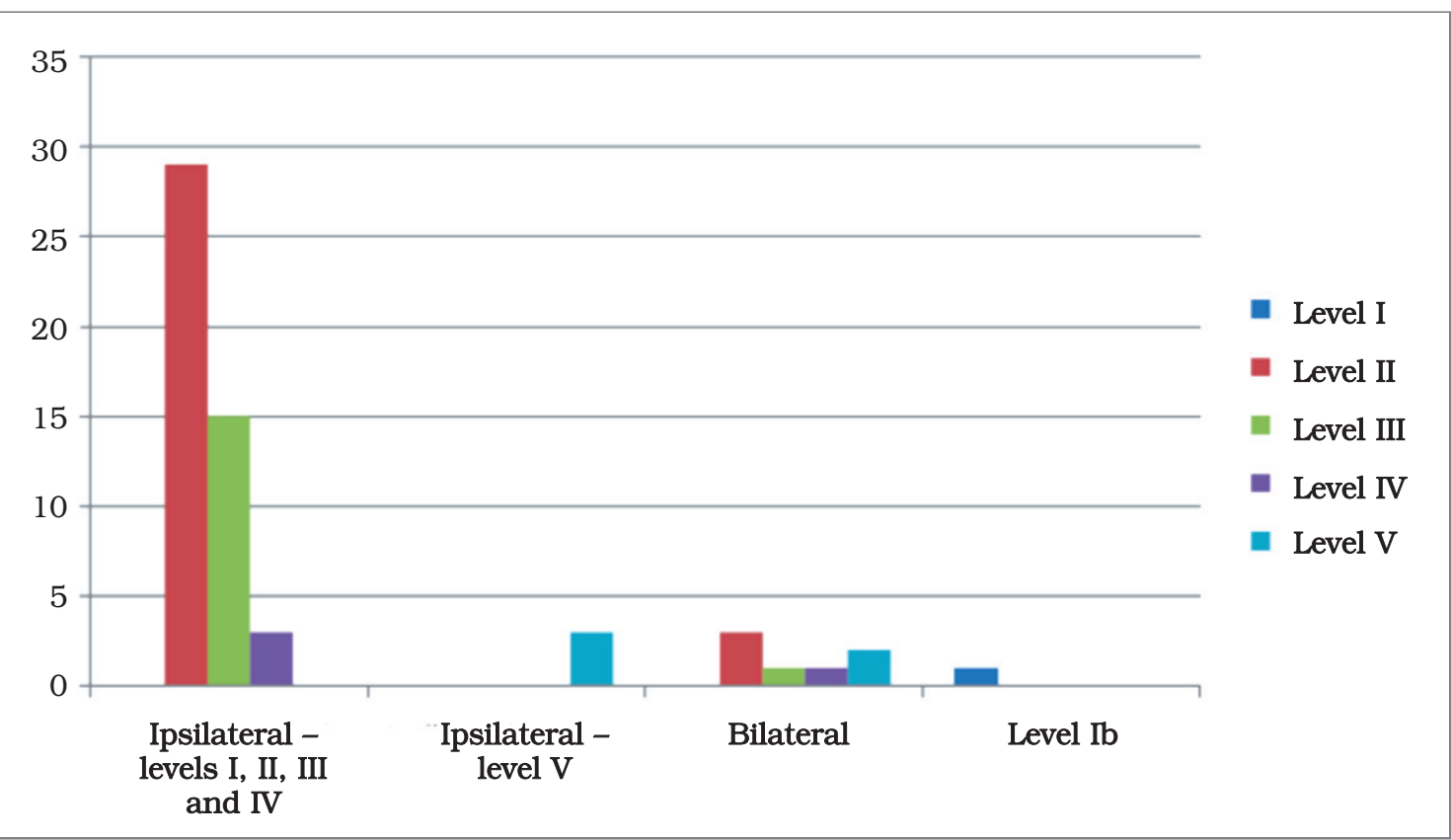

Fig. 4 Nodal MTS in oro-, and hypopharyngeal cancer

3) In the third group, we had 16 patients with laryngeal cancer, of which 2 were glottic and 14 supraglottic. In all patients there were nodal metastases in the predilected lymphatic area.

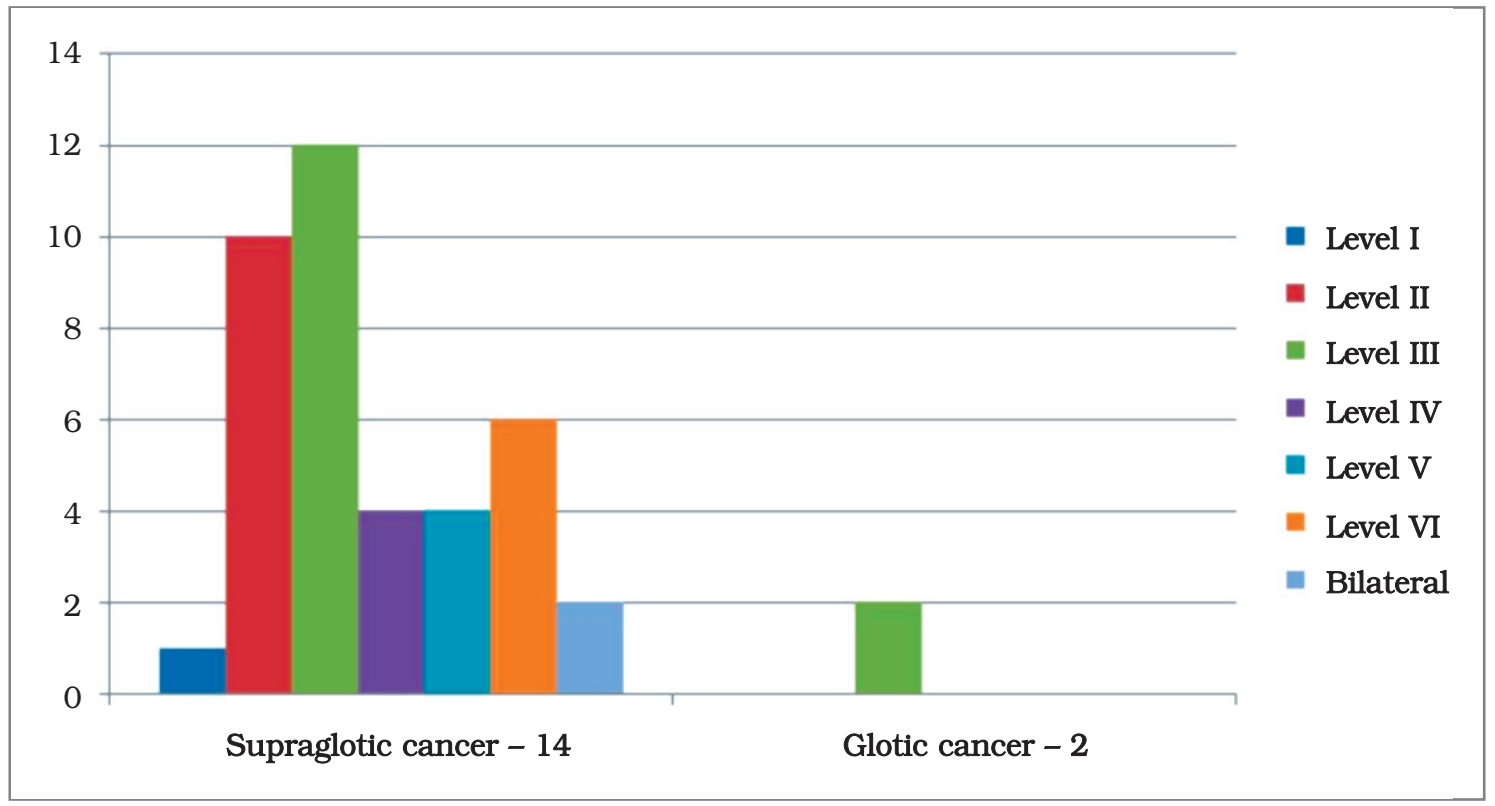

Fig.5 Nodal MTS in laryngeal cancer 


\section{DISCUSSION AND CONCLUSIONS}

Based on literature and practice we know that the occurrence of nodal metastases is one of the most important prognostic factors in head and neck cancer. Early diagnosis is crucial for proper treatment. Unfortunately, the detection of early metastases remains a major challenge despite the constant development of imaging modalities (7). Whereas predictive diagnostic methods have they own limitation, at Clinic we decided to prefer a selective neck dissection based on a surgical treatment of predilected lymphatic spread area for each type of head and neck tumor individually with a preservation of non-lymphatic structures of the neck.

To confirm the correctness of our practice and to question the sentinel lymph node method we did a retrospective study.

We evaluated patients who had been treated at our Clinic for an oncologic disease and who had undergone a neck block dissection and who had a nodal metastasis of the primary tumor confirmed.

The main goal was to analyze the distribution of neck metastases and to study the frequency of skip metastases in head and neck cancer.

In our group of patients we confirmed a skip metastasis in $2.1 \%$ of cases $(3.3 \%$ in pharyngeal carcinomas). The low incidence in our cohort was probably caused by an inaccurate separation of the dissected adipo-lymphatic tissue according to the levels I-V during the surgery or, in the case of extensive N3 metastases, the inability to exactly determine in which group the original lymph nodes were present.

Also the size of the group of patients itself is a possible cause of the low incidence of skip metastases.

At present we have adopted precise rules according to which the separation of dissected adipo-lymphatic tissue in elective and therapeutic cervical dissections will proceed in the future. After a sufficient enlargement of the group of patients we will evaluate the occurrence of skip metastases again.

\section{REFERENCES}

1. Čelakovský P, Plzák J, Betka J, et al. Krční metastázy, Tobiáš 2012

2. Klener P. Základy klinické onkologie, Praha: Galén/Karolinum, 2002

3. Rivière D, Mancini J, Santini L, Giovanni A, DessiP, Fakhry N. Lymph-node metastasis following total laryngectomy and total pharyngolaryngectomy for laryngeal and hypopharyngeal squamous cell carcinoma: Frequency,distribution and risk factors. European Annals of Otorhinolaryngology, Head and Neck diseases 2018; 135 (3): 163166.

4. Bhuiyan MR, Ali MI, Siddiquee B, Johora F, Hossain MK. Frequency of Lymph node Metastasis in Supraglottic Ca Larynx.Medicine Today 2019; 31(2): 72-75.

5. Novotný J, Vítek P, Kleibl Z, et al. Onkologie v klinické praxi. 3rd ed. Praha: Mladá fronta; 2019. p. 114-142

6. Cariati P, Serrano CA, Solis FJ, Martinez Lara I. Distribution of cervical metastasis in tongue cancer: Are occult metastases predictable? A retrospective study of 117 oral tongue carcinomas, Journal of Cranio-Maxillofacial Surgery 2017. 46 (1): 155-161.

7. Forghani R, Chatterjee A, Reinhold C, et al. Head and neck squamous cell carcinoma: prediction of cervical lymph node metastasis by dual-energy CT texture analysis with machine learning. European Radiology 2019. 29:6172-6181.

8. Demir D. The Role of Sentinel Lymph Node Biopsy in Head and Neck Cancers and Its Application Areas, Turkish Archives of Otorhinolaryngology 2016; 54: 35-38

9. Shikharani P, Ishwar S, Achal G, Nita K. A Study on Neck Nodes in Oral Cancers, with Special Reference to Skip Metastasis. Indian Journal of Otolaryngology and Head \& Neck Surgery 2018; 71: 474-481. 
10. Gray H, Lewis WH. Anatomy of the Human Body, 20th Edition. Lea \& Fiber 1918.

11. Haksever M, Yurtaslan A, Inancli HM, Tuncel Ü, Kürkcüoglu SS, Uyar M, Gene Ö, Irkkan C. The Effects of Tumor Size, Degree of Differentiation, and Depth of Invasion on the risk of Neck Node Metastasis in Squamous Cell carcinoma of the Oral Cavity. Ear, Nose \& Throat Journal 2012; 91(3):130-135.

12. Lim SC, Zhang S, Ishii G, et al. Predictive markers for late cervical metastasis in stage I and II invasive squamous cell carcinoma of the oral tongue. Clinical CancerResearch 2004; 10(1):166-72.

Received: November, 11, 2020

Accepted: June, 30, 2021 